\title{
Multifocal insulinoma secondary to insulinomatosis: persistent hypoglycaemia despite total pancreatectomy
}

\author{
Jennifer R Snaith1,2,3,4, Duncan McLeod4,5,6, Arthur Richardson4,7,8 and David Chipps 1,4 \\ 1Department of Diabetes and Endocrinology, Westmead Hospital, Westmead, New South Wales, Australia, \\ 2Department of Diabetes and Endocrinology, St Vincent's Hospital, Darlinghurst, New South Wales, Australia, \\ ${ }^{3}$ Healthy Ageing, Garvan Institute of Medical Research, Darlinghurst, New South Wales, Australia, ${ }^{4}$ Sydney Medical \\ School, University of Sydney, Sydney, New South Wales, Australia, ${ }^{5}$ Department of Anatomical Pathology, Institute of \\ Clinical Pathology and Medical Research, Westmead Hospital, Sydney, New South Wales, Australia, ${ }^{6}$ Westmead \\ Institute for Medical Research, Sydney, New South Wales, Australia, 7Department of Surgery, Westmead Hospital, \\ Sydney, New South Wales, Australia, and ${ }^{8}$ Department of Surgery, Sydney Adventist Hospital, Sydney, New South \\ Wales, Australia
}

Correspondence should be addressed to J R Snaith Email jennifer.snaith@health.nsw. gov.au

\section{Summary}

Insulinomatosis is a rare cause of hyperinsulinaemic hypoglycaemia. The ideal management approach is not known. A 40-year-old woman with recurrent symptomatic hyperinsulinaemic hypoglycaemia was diagnosed with an insulinoma. A benign $12 \mathrm{~mm}$ pancreatic head insulinoma was resected but hypoglycaemia recurred 7 years later. A benign $10 \mathrm{~mm}$ pancreatic head insulinoma was then resected but hypoglycaemia recurred within 2 months. Octreotide injections were trialled but exacerbated hypoglycaemia. After a 2-year interval, she underwent total pancreatectomy. A benign $28 \mathrm{~mm}$ pancreatic head insulinoma was found alongside insulin-expressing monohormonal endocrine cell clusters (IMECCS) and islet cell hyperplasia, consistent with a diagnosis of insulinomatosis. Hypoglycaemia recurred within 6 weeks. There was no identifiable lesion on MRI pancreas, Ga-68 PET or FDG PET. Diazoxide and everolimus were not tolerated. MEN-1 testing was negative. Insulinomatosis should be suspected in insulinomas with early recurrence or multifocality. De novo lesions can arise throughout the pancreas. Extensive surgery will assist diagnosis but may not provide cure.

\section{Learning points:}

- Insulinomas are usually benign and managed surgically.

- Insulinomatosis is characterised by multifocal benign insulinomas with a tendency to recur early. It is rare.

- Multifocal or recurrent insulinomas should raise suspicion of MEN-1 syndrome, or insulinomatosis.

- Insulinomatosis is distinguished histologically by insulin-expressing monohormonal endocrine cell clusters (IMECCS) and tumour staining only for insulin, whereas MEN-1 associated insulinomas stain for multiple hormones.

- The ideal treatment strategy is unknown. Total pancreatectomy may not offer cure.

\section{Background}

Insulinomas are pancreatic neuroendocrine tumours that cause hypoglycaemia by excessive insulin secretion (1). These tumours are typically benign, solitary and surgically cured $(2,3)$.

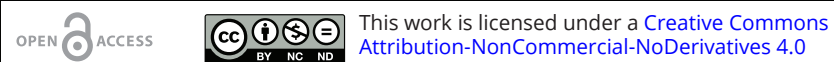
International License.
}

Insulinomas usually occur sporadically or in association with multiple endocrine neoplasia (MEN1) syndrome. MEN-1 associated insulinomas tend to be multifocal and recur (4). Recurrence risk is up to $21 \%$ at 
10 years, compared to $5 \%$ without MEN- 1 syndrome $(3,5)$. Given these important differences, the surgical approach to MEN-1 associated insulinomas involves subtotal pancreatectomy rather than lesion-only enucleation (6).

Insulinomatosis is a cause of multifocal insulinomas without MEN-1 syndrome. It is typified by insulinomas with concurrent insulin-expressing monohormonal endocrine cell clusters (IMECCs). IMECCs may represent tumour precursor lesions. Compared to MEN-1 associated insulinomas, insulinomatosis has greater relapse potential ( $46 \%$ vs $31 \%$ ) that occurs earlier (mean relapse 8 vs 14 years) $(5,7)$. Histologically, insulinomatosis is distinguished by tumour staining only for insulin and by IMECCs, whereas MEN-1 associated insulinomas stain for multiple hormones $(5,7)$. Notably, a case series describing multifocal insulinomas reported hyperinsulinaemic hypoglycaemia in MEN-1 syndrome only with lesions larger than $5 \mathrm{~mm}$ (macroadenomas), yet hypoglycaemia with lesions less than $5 \mathrm{~mm}$ (microadenomas) in nonMEN-1 associated multifocal insulinomas (7). This implies that in insulinomatosis, even small lesions may be functional.

In insulinomatosis, propensity for tumourigenesis may be related to dysregulation of the MENIN-MAFA pathway. MENIN controls beta cell differentiation and proliferation via the production of MAFA (V-Maf avian musculoaponeurotic fibrosarcoma oncogene homolog A), a beta cell transcription factor (8). A mutation in MAFA has been associated with familial forms of insulinomatosis but not in non-familial cases. Presumably the disease is mediated by an aberrant beta cell differentiation factor affecting the entire beta cell population. This implies a lifelong recurrence risk unless the pancreas is completely removed. We describe the first report of insulinomatosis with persistent hypoglycaemia despite total pancreatectomy. This case captures a typically long investigative course before diagnosis and highlights that aggressive surgical treatment may not be curative.

\section{Case presentation}

A 40-year-old previously well female presented with 4 years of agitation exacerbated by fasting and improved by food ingestion. Blood tests performed at the time of a seizure identified a blood glucose level of $2.0 \mathrm{mmol} / \mathrm{L}$, C-peptide $515 \mathrm{pmol} / \mathrm{L}$ (365-1655 pmol/L) and insulin 5.9 $\mathrm{mIU} / \mathrm{L}$ (fasting level $<16.0 \mathrm{mIU} / \mathrm{L}$ ). There was no family history of endocrine neoplasia or diabetes.

Abdominal CT detected a $12 \mathrm{~mm}$ mass in the body of the pancreas. A benign cystadenoma was resected, but hypoglycaemia persisted and she was referred to our service. Endogenous fasting hyperinsulinaemia was again confirmed (plasma glucose $1.6 \mathrm{mmol} / \mathrm{L}$, serum insulin $44 \mathrm{mIU} / \mathrm{L}, \mathrm{C}$-peptide $1.76 \mathrm{nmol} / \mathrm{L}$ and proinsulin 122 $\mathrm{pmol} / \mathrm{L})$. Sulfonylurea screen was negative. An $8 \mathrm{~mm}$ head of pancreas lesion was detected by MRI and an uncinate lesion by endoscopic ultrasound (EUS). There was no focal uptake on Indium-111 Octreotide scanning, and selective arterial calcium stimulation testing (SACST) into feeding vessels of the pancreas did not provoke insulin secretion in any supply territory.

A benign $12 \mathrm{~mm}$ pancreas head insulinoma was removed with a second laparotomy. The tumour stained for insulin (negative for glucagon and somatostatin). She was without hypoglycaemia for 2 months but did not return for review.

After 7 years, she represented with hyperinsulinaemic hypoglycaemia redemonstrated on a supervised fast. Diazoxide therapy was ineffective. MRI detected a $13 \mathrm{~mm}$ liver lesion but Gallium-68 PET and EUS were unremarkable. SACST regionalised to the pancreas head, indicated by a doubling of hepatic vein insulin following calcium injection into the gastroduodenal and superior mesenteric arteries. A $10 \mathrm{~mm}$ benign insulinoma was resected from the pancreas head at a third laparotomy. Ki 67 was less than 1\%, and the tumour stained monohormonally for insulin. The liver lesion was focal nodular hyperplasia. Her hypoglycaemia again resolved postoperatively.

However, hypoglycaemia recurred within 2 months. MRI and Gallium-68 PET were unremarkable. Octreotide injections exacerbated hypoglycaemia and the patient did not return for review for another 2 years. Repeat abdominal MRI and EUS again did not demonstrate a lesion, but SACST revealed a doubling of hepatic vein insulin concentrations following calcium injection into the gastroduodenal, superior mesenteric and hepatic arteries, but not after calcium injection into the splenic artery, indicating regionalisation to the head of pancreas and liver. A total pancreatectomy was then performed including pancreaticoduodenectomy, removal of the body and tail of the pancreas, splenectomy and cholecystectomy. It was surgically difficult with pancreas adherent to retroperitoneum.

\section{Investigation}

Insulinomatosis was diagnosed with a $28 \mathrm{~mm}$ welldifferentiated head of pancreas insulinoma (Ki-67 $3.3 \%$, without lymphovascular invasion) (Fig. 1), a 


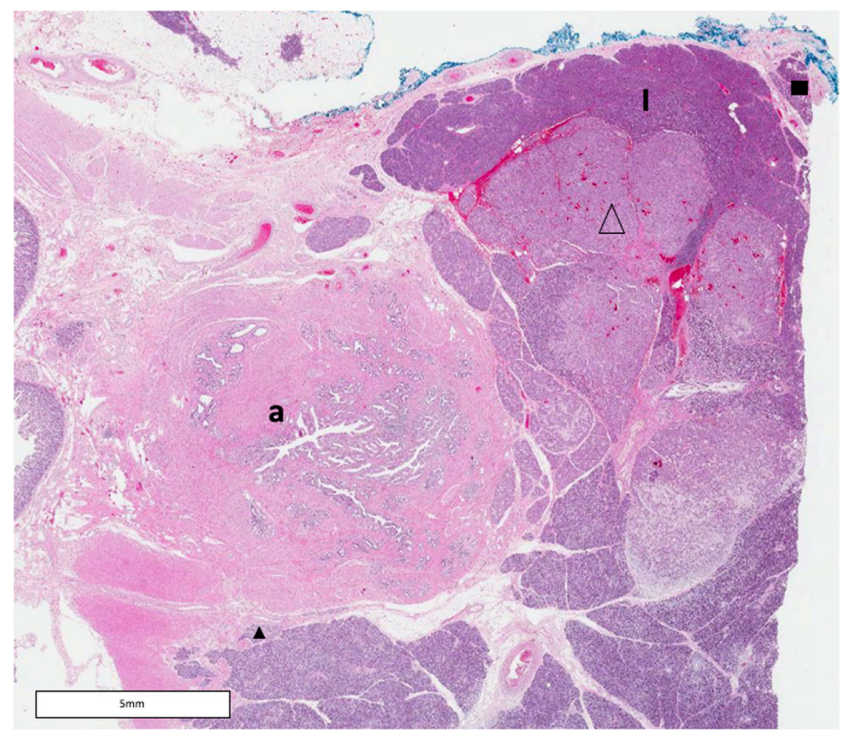

\section{Figure 1}

Low power view of head of pancreas demonstrating insulinoma $(\Delta)$, microadenoma $(\boldsymbol{\square})$, insulin expressing monohormonal cell cluster $(\boldsymbol{\Delta}$ normal islets (I) and ampulla of Vater (a).

$2 \mathrm{~mm}$ microadenoma that stained monohormonally for insulin (Fig. 2), numerous IMECCs and islet hyperplasia throughout the pancreas. MEN-1 and MAFA mutation testing was negative (D. Iacovazzo, personal communication).

\section{Treatment}

After total pancreatectomy, she commenced subcutaneous insulin and pancrelipase supplementation but did not attend follow up. Insulin injections were ceased after 6 weeks, and hypoglycaemia recurred. A random C-peptide level of $6.8 \mathrm{nmol} / \mathrm{L}$ confirmed ongoing endogenous insulin secretion. CT, MRI, Gallium-68 PET and FDG PET failed to identify a lesion. She was treated with everolimus $10 \mathrm{mg}$ daily for 2 months but this was ceased after recurrent cellulitis and lack of efficacy.

\section{Outcome and follow-up}

Hypoglycaemia continues to persist. She continues to consume carbohydrates every two hours avoid hypoglycaemia.

\section{Discussion}

This is the first report of insulinomatosis with failed cure by total pancreatectomy. Total pancreatectomy was performed given the patient's history of tumour

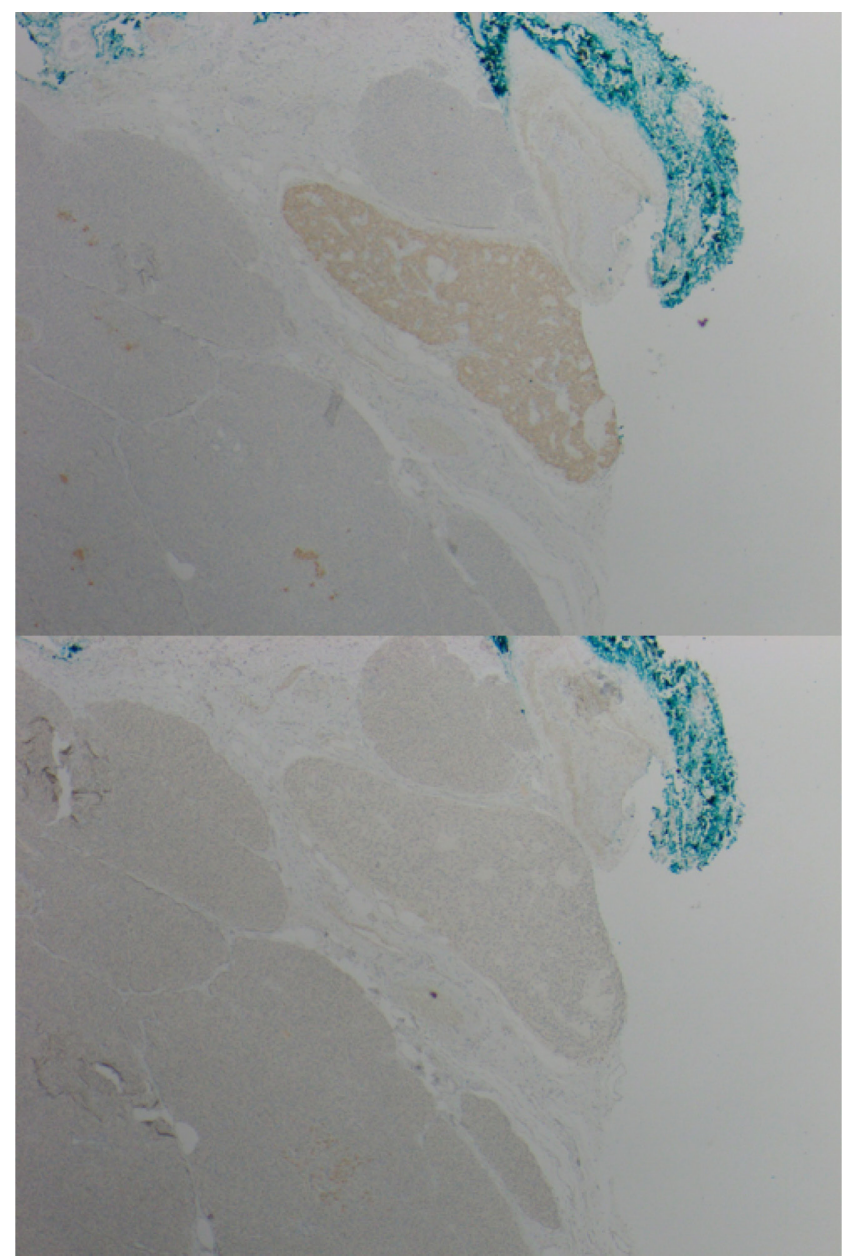

\section{Figure 2}

Immunohistochemistry staining shows positive staining for insulin (upper panel), and negative staining for glucagon (lower panel).

recurrence, and the technical difficulty of adhesions from prior operations precluding further surgery. The diagnosis of insulinomatosis was not suspected prior to the total pancreatectomy but was only revealed by the findings of IMECCs and hyperplasia throughout the pancreas. Therefore, the extensive surgery established the diagnosis and provided potential cure.

Surgical management of an insulinoma may vary if a causative syndrome is suspected. In sporadic insulinoma, enucleation usually provides cure and preservation of healthy pancreatic parenchyma (2). The ideal surgical approach in MEN-1 associated insulinomas is debated. Given the higher risk of recurrence, multifocality and malignancy, subtotal pancreatic resection rather than enucleation has been advocated (4). Whether more extensive resection reduces recurrence risk or has an impact on survival remains unclear. Consensus guidelines also endorse radical surgery if recurrence or malignancy is 
suspected (1). The optimal approach in insulinomatosis is unknown.

There is one other report of insulinomatosis managed with complete resection of the pancreas. This case involved a 43-year-old female with a protracted investigative course and 3 pancreas operations. Insulinomatosis was diagnosed histologically following her second operation, but persisting hypoglycaemia was managed by resection of the remnant pancreas (9). Whether hypoglycaemia persisted postoperatively or recurred was not reported. Our case was also similarly managed with multiple sequential surgeries leading to total pancreatectomy, but hypoglycaemia recurred. Therefore, we report the first case of failure to cure insulinomatosis by total pancreatectomy. Other reported cases managed with enucleation, partial pancreatic resection, or Whipple's procedure report variable relapse rates (5). In a case series of 14 patients, 8 were successfully managed with a single operation: distal pancreatectomy (one patient also had enucleation of a tumour from the residual pancreas during the same operation). One patient had recurrent hypoglycaemia following distal pancreatectomy but died within 12 months. The cause of death was not reported. Multiple operations were performed in 5/14 patients to manage recurrent hypoglycaemia. Thirteen patients in this case series remained symptom-free after their final operation (data not available for 1/14). Whilst the follow up duration was not described, these data suggests that surgery can potentially offer cure. The poor outcome in our case may relate to difficult dissection of the remnant pancreas leaving microscopic residual tissue. Our case supports more extensive surgery in establishing the diagnosis of insulinomatosis, yet total pancreatectomy may not necessarily provide cure, especially if complicated by adhesions from multiple reoperations.

In this patient, successive recurrences occurred 7 years, 2 months, then 2 years after each tumour resection. Thus, despite benign histology, insulinomatosis tumours can display aggressive growth potential. The early recurrence presumably results from de novo primary lesions, rather than loco-regional recurrence (5). This is supported by the finding of synchronous lesions across multiple areas of the pancreas. We postulate that new lesion growth following total pancreatectomy arose from microscopic residual pancreatic tissue in the surgical bed that was unresectable from surrounding fibrous tissue after multiple operations.

Micro-metastases cannot be excluded. The doubling of hepatic vein insulin during SACST was suggestive of liver metastases. At the time, this result was considered to be falsely positive due to retrograde calcium flow from the hepatic into the gastro-duodenal artery supplying a lesion in the pancreatic head and was supported by the apparent resolution of hypoglycaemia after surgical removal of the pancreatic head lesion. Metastasis in insulinomatosis is rare, with reports of only one case with lymph node and liver metastasis (5). As microadenomas are capable of a producing hypoglycaemia, it is feasible that occult metastases could account for persistent hypoglycaemia in our patient, and that these lesions were smaller than the threshold of detection by available imaging modalities.

Localisation of insulinoma lesions is difficult, and often requires multiple modalities. In our patient, crosssectional imaging failed to detect a $28 \mathrm{~mm}$ pancreatic lesion. Functional lesions were most reliably detected with SACST, a test of tumour secretory capacity. PET imaging with exendin-4 analogues was not available at our institution but has been used in one case report of insulinomatosis (10), in which 68-Gallium-DOTAExendin PET/CT and MRI detected lesions up to $5 \mathrm{~mm}$ in size but failed to detect multiple microadenomas identified histologically. GLP-1 receptor expression in insulinomatosis has otherwise not been characterised.

Finally, our patient's tumour immunostaining patterns provided possible early clues to the presence of insulinomatosis. Each tumour stained for insulin, but not for other hormones including glucagon and somatostatin. Monohormonality was described by Anlauf et al. as one of the hallmark features that distinguish insulinomatosis from a MEN-1 associated tumours (5). However, this feature does not distinguish insulinomatosis from sporadic benign insulinomas which stain monohormonally in $73 \%$ of cases (11). The pattern of early recurrence and multifocality was suggestive of insulinomatosis, but the diagnosis was only confirmed by the finding of IMECCs at the time of her final operation.

In conclusion, insulinomatosis is a rare cause of multifocal insulinoma and should be considered in early recurrent insulinomas, or multifocal insulinomas without MEN-1 syndrome that stain only for insulin hormone. More extensive pancreas resection is required for diagnosis but may be therapeutically ineffective. The presented case contributes significantly to our understanding of this condition. It highlights the high recurrence risk and treatment limitations. Total pancreatectomy may not be curative as hypoglycaemia may occur from unresectable microscopic functional lesions, or occult metastases.

\section{Declaration of interest}

The authors declare that there is no conflict of interest that could be perceived as prejudicing the impartiality of the research reported. 


\section{Funding}

This research did not receive any specific grant from any funding agency in the public, commercial or not-for-profit sector.

\section{Patient consent}

Written informed consent for publication of their clinical details and images was obtained from the patient.

\section{Author contribution statement}

J Snaith prepared the first version of the manuscript. D McLeod provided the figures and expert opinion on review of pathology specimens. J Snaith, D Chipps and A Richardson contributed to the management of the patient. All authors reviewed the manuscript.

\section{References}

1 Jensen RT, Cadiot G, Brandi ML, de Herder WW, Kaltsas G, Komminoth P, Scoazec JY, Salazar R, Sauvanet A, Kianmanesh R, et al. Enets consensus guidelines for the management of patients with digestive neuroendocrine neoplasms: functional pancreatic endocrine tumor syndromes. Neuroendocrinology 201295 98-119. (https://doi.org/10.1159/000335591)

2 Crippa S, Zerbi A, Boninsegna L, Capitanio V, Partelli S, Balzano G, Pederzoli P, Di Carlo V \& Falconi M. Surgical management of insulinomas: short- and long-term outcomes after enucleations and pancreatic resections. Archives of Surgery 2012147 261-266. (https:// doi.org/10.1001/archsurg.2011.1843)

3 Service FJ, McMahon MM, O’Brien PC \& Ballard DJ. Functioning insulinoma - incidence, recurrence, and long-term survival of patients: a 60-year study. Mayo Clinic Proceedings 199166 711-719. (https://doi.org/10.1016/s0025-6196(12)62083-7)
4 Shin JJ, Gorden P \& Libutti SK. Insulinoma: pathophysiology, localization and management. Future Oncology 20106 229-237. (https://doi.org/10.2217/fon.09.165)

5 Anlauf M, Bauersfeld J, Raffel A, Koch CA, Henopp T, Alkatout I, Schmitt A, Weber A, Kruse ML, Braunstein S, et al. Insulinomatosis: a multicentric insulinoma disease that frequently causes early recurrent hyperinsulinemic hypoglycemia. American Journal of Surgical Pathology 200933 339-346. (https://doi.org/10.1097/ PAS.0b013e3181874eca)

6 Nikfarjam M, Warshaw AL, Axelrod L, Deshpande V, Thayer SP, Ferrone CR \& Fernández-del Castillo C. Improved contemporary surgical management of insulinomas: a 25-year experience at the Massachusetts General Hospital. Annals of Surgery 2008247 165-172. (https://doi.org/10.1097/SLA.0b013e31815792ed)

7 Anlauf M, Schlenger R, Perren A, Bauersfeld J, Koch CA, Dralle H, Raffel A, Knoefel WT, Weihe E, Ruszniewski P, et al. Microadenomatosis of the endocrine pancreas in patients with and without the multiple endocrine neoplasia Type 1 syndrome. American Journal of Surgical Pathology 200630 560-574. (https://doi. org/10.1097/01.pas.0000194044.01104.25)

8 Iacovazzo D, Flanagan SE, Walker E, Quezado R, de Sousa Barros FA, Caswell R, Johnson MB, Wakeling M, Brändle $\mathrm{M}$, Guo $\mathrm{M}$, et al. MAFA missense mutation causes familial insulinomatosis and diabetes mellitus. PNAS 2018115 1027-1032. (https://doi.org/10.1073/ pnas.1712262115)

9 Guojing Y, Jingtao D, Li Z \& Yiming M. A Special form of Pancreatic Hyperinsulinemic Hypoglycaemia - Insulinomatosis: A Case Report. Endocrine Reviews 201435 i1-i1153. (https://doi.org/10.1093/ edrv/35.supp.1)

10 Jenni S, Antwi K, Fani M, Wild D, Heye T, Gloor B, et al. Multifocal insulinomas (insulinomatosis) in GLP-1-receptor PET/CT. In Presented at the 17th European Congress of Endocrinology, May 16-20. Dublin, Ireland: BioScientifica, 2015. (https://doi.org/10.1530/endoabs.37.EP1115)

11 Kapran Y, Bauersfeld J, Anlauf M, Sipos B \& Klöppel G. Multihormonality and entrapment of islets in pancreatic endocrine tumors. Virchows Archiv 2006448 394-398. (https://doi.org/10.1007/ s00428-005-0147-4)

Received in final form 4 September 2020 Accepted 30 October 2020 